\title{
Non-Coherent Cooperative Communications Dispensing with Channel Estimation Relying on Erasure Insertion Aided Reed-Solomon Coded SFH M-ary FSK Subjected to Partial-Band Interference and Rayleigh Fading
}

\author{
Hoang Anh Ngo, Student, IEEE, Sohail Ahmed, Lie-Liang Yang, Senior Member, IEEE, \\ and Lajos Hanzo, Fellow, IEEE
}

\begin{abstract}
The rationale of our design is that although much of the literature of cooperative systems assumes perfect coherent detection, the assumption of having any channel estimates at the relays imposes an unreasonable burden on the relay station. Hence, non-coherently detected Reed-Solomon (ReS) coded Slow Frequency Hopping (SFH) assisted $M$-ary Frequency Shift Keying (FSK) is proposed for cooperative wireless networks, subjected to both partial-band interference and Rayleigh fading. Erasure insertion (EI) assisted ReS decoding based on the joint maximum output-ratio threshold test (MO-RTT) is investigated in order to evaluate the attainable system performance. Compared to the conventional error-correction-only decoder, the EI scheme may achieve an $E_{b} / N_{0}$ gain of approximately 3 dB at the Codeword Error Probability, $P_{w}$, of $10^{-4}$, when employing the $\operatorname{Re} S(31,20)$ code combined with 32-FSK modulation. Additionally, we evaluated the system's performance, when either equal gain combining (EGC) or selection combining (SC) techniques are employed at the destination's receiver. The results demonstrated that in the presence of one and two assisting relays, the EGC scheme achieves gains of $1.5 \mathrm{~dB}$ and $1.0 \mathrm{~dB}$ at the $P_{w}$ of $10^{-6}$, respectively, compared to the SC arrangement. Furthermore, we demonstrated that for the same coding rate and packet size, the $\operatorname{Re} S(31,20)$ code using EI decoding is capable of outperforming convolutional coding, when 32-FSK modulation is considered, whilst LDPC coding had an edge over the above two schemes.
\end{abstract}

Index Terms-Slow frequency hopping, Reed-Solomon codes, frequency shift keying, erasure insertion decoding, the joint maximum output-ratio threshold test, equal gain combining, selection combining.

\section{INTRODUCTION}

O WING to the ability of improving the achievable performance, the family of Multiple-Input-MultipleOutput (MIMO) techniques [1], [2] has been adopted for

Paper approved by M.-S. Alouini, the Editor for Modulation and Diversity Systems of the IEEE Communications Society. Manuscript received October 8, 2010; revised June 10 and October 28, 2011.

The authors are with the School of Electronics and Computer Science, University of Southampton, Southampton, SO17 1BJ, UK (e-mail: \{han08r, lh\}@ecs.soton.ac.uk).

The research leading to these results has received funding from the European Union's Seventh Framework Programme ([FP7/2007-2013]) under the auspices of the Concerto project; the financial support of RC-UK under the auspices of the UK-India Advanced Technology Centre; as well as that of the China-UK Science Bridge in $4 \mathrm{G}$ Wireless Communications is also gratefully acknowledged.

Digital Object Identifier 10.1109/TCOMM.2012.061112.100616 various wireless standards [3]. However, it is ineffective to employ multiple antennas in mobile handsets, since their diversity gain remains limited by the spatial correlation imposed by their compact physical dimensions. In order to overcome this obstacle, relaying techniques were proposed [2], [4], [5], where single-antenna aided mobile stations collaborate to create a distributed MIMO system.

The rationale of our design is that although much of the literature of cooperative systems assumes perfect coherent detection, the assumption of having any channel estimates at the relays imposes an unreasonable burden on the relay station. Hence, in this contribution we investigate low-complexity non-coherent detection aided relaying in the absence of channel information. In Slow Frequency Hopping (SFH) assisted $M$-ary Frequency Shift Keying (FSK) employing ReedSolomon ( $\mathrm{ReS}$ ) coding, typically error-and-erasure decoding is utilized in order to improve the attainable system performance, when subjected to interference, jamming and fading [6]-[8]. Several erasure insertion (EI) techniques assisting error-anderasure ReS decoding were proposed in [6], [9], namely the ratio-threshold test (RTT), the output threshold test (OTT) and the joint maximum output and ratio threshold test (MO-RTT). However, none of these techniques have been investigated in the context of distributed MIMO systems.

Against this backcloth, in this contribution we propose a non-coherently detected ReS coded SFH M-FSK system, dispensing with any channel information, subjected to partialband interference and Rayleigh fading, in the context of wireless cooperative networks. The EI technique will be invoked for error-and-erasure ReS decoding based on the joint MO-RTT arrangement, along with the detailed analysis of the related decision statistics. With the aid of these decision statistics, the analytical expressions of the $\operatorname{ReS}$ codeword error probability as well as the bit error probability (BEP) achieved by erasure decoding will be derived. Our results show that in the context of the wireless relaying network, the EI ReS decoding scheme may significantly enhance the overall achievable system performance.

Furthermore, we compared the performance of the proposed $\mathrm{ReS}$ coded system in wireless cooperative networks to those of convolutional codes and Low Density Parity Check (LDPC) 


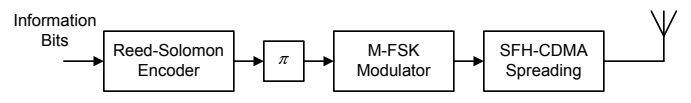

Fig. 1. The transmitter block diagram of the Source Station using ReedSolomon coded SFH $M$-ary FSK.

codes [10], which support efficient iterative detection at the cost of relying on sophisticated transceiver designs. Our results demonstrated that although the proposed ReS coding scheme is capable of outperforming convoluational codes in the context of short-packet transmissions, the LDPC code considered had an edge. Therefore, the ReS coded systems using errorand-erasure decoding strikes an tractive tradeoff for a system requiring low complexity as well as low latency.

The outline of this paper is as follows. Section II describes the system's structure and the associated assumptions. The MO-RTT EI scheme and the related decision statistics are considered in Section III, followed by our numerical results in Section IV. Finally, our concluding remarks are offered in Section V.

\section{SYSTEM DESCRIPTION}

The system under consideration consists of a source station (SS), $L$ relay stations (RS) and a destination station (DS) communicating over Rayleigh fading channels subjected to Partial-Band Gaussian Interference (PBGI). The communication process is divided into two time slots. In the first slot, the SS broadcasts its packets to all the RSs and to the DS. In the second slot, each RS will forward the re-encoded packets to the DS, if it correctly decoded the received information. Otherwise, the packet will be ignored at the RS.

The SS's transmitter structure is shown in Fig. 1. At the SS, the data bits are first encoded by the $(N, K)$ ReS code defined over the Galois field $G F\left(2^{b}\right)$, which turns $K b$-bit uncoded symbols into $N$ ReS-encoded symbols. Subsequently, the encoded symbols are interleaved and passed to the $M$-ary FSK modulator. We assume that $M=N=2^{b} \geq 4$, so that each $b$-bit ReS coded symbol describes an $M$-ary FSK symbol. Finally, the frequency synthesizer, which operates under the control of a pseudo noise (PN) generator, generates a sequence of random hopping frequencies, one of which is activated during each hop interval of duration $T_{h}$, or symbol interval $T_{s}$, where we assume $T_{h}=T_{s}$. In order to allow non-coherent detection at the receiver, the bandwidth of a single frequency hopping $(\mathrm{FH})$ tone is given by $B=1 / T_{h}$. The transmit signal at the SS may be modelled as

$$
x_{i}(t)=\sqrt{2 E_{s} R_{c}} \cos \left\{2 \pi\left(f_{n}+f_{i}\right) t+\varphi_{n}+\varphi_{i}\right\},
$$

where $E_{s}$ is the symbol power at the SS, $R_{c}=K / N$ is the ReS code-rate; $f_{n}$ is the hopping frequency during the $n^{\text {th }}$ FH interval and $f_{i}$ is the $i^{t h}$ frequency associated with the $i^{t h}$ transmitted MFSK/ReS symbol. Finally, $\varphi_{n}$ and $\varphi_{i}$ are random phases during the $n^{\text {th }} \mathrm{FH}$ interval and the $i^{\text {th }}$ symbol interval.

The modulated signal of each FH tone is transmitted over the frequency-flat fading channels obeying the Rayleigh dis-

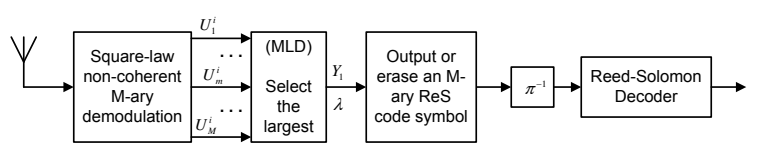

Fig. 2. The receiver block diagram of the Relay Station using Reed-Solomon coded SFH $M$-ary FSK.

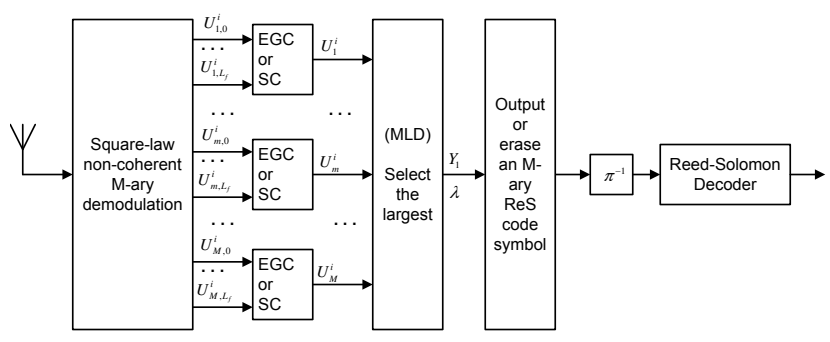

Fig. 3. The receiver block diagram of the Destination Station using ReedSolomon coded SFH $M$-ary FSK.

tribution having the probability density function (p.d.f) given by

$$
f(|h|)=\frac{2|h|}{\Omega} e^{-|h|^{2} / \Omega}
$$

where we have $\Omega=E\left[|h|^{2}\right]$.

Furthermore, the communication channels are assumed to be contaminated by both PBGI and additive white Gaussian noise (AWGN). The PBGI occupies a fraction of $\rho \leq 1$ band, having the power spectral density of $N_{I} / 2 \rho$, while the AWGN has the power spectral density of $N_{0} / 2$. Consequently, in the specific portion of the band interfered by the PBGI, the total noise power spectral density is $N_{n} / 2=N_{0} / 2+N_{I} / 2$, while it is $N_{n} / 2=N_{0} / 2$ in the remainder of the band.

As a result, the signal received at the $l^{\text {th }}$ RS may be expressed as

$r_{i l}(t)=G_{s r_{l}} h_{i, s r_{l}} \sqrt{2 E_{s} R_{c}} \cos \left\{2 \pi\left(f_{n}+f_{i}\right) t+\phi_{i, s r_{l}}\right\}+n_{i, s r_{l}}(t)$,

where $G_{s r}$ is the pathloss-reduction related gain [11], [12], $h_{i, s r_{l}}$ is the fading coefficient of the $l^{t h}$ Source-to-Relay (SR) link, $n_{i, s r_{l}}$ is the effective noise as defined above and $\phi_{i, s r_{l}}$ includes all the phases in the received signal due to frequency hopping, carrier- and MFSK-modulation as well as that induced by the fading.

After demodulation, the RS will demodulate and ReSdecode the packet. If the packet is correctly ReS-decoded, it will be re-encoded, re-modulated and forwarded to the DS. Otherwise, the RS will ignore the packet and turn to idle mode, waiting for a new packet to arrive from the SS. The probability of a packet being forwarded from the RS to the DS will be detailed in Section III. The RS's receiver block diagram is shown in Fig. 2 while its transmitter is similar to the SS's transmitter.

Finally, the signal received at the DS may be expressed as 


$$
y_{i}(t)=\sum_{l=0}^{L} G_{l} h_{i, l} \sqrt{2 E_{l} R_{c}} \cos \left\{2 \pi\left(f_{n}+f_{i}\right) t+\phi_{i, l}\right\}+n_{i, l}(t)
$$

where $G_{l}$ is the pathloss-reduction related gain of the $l^{\text {th }}$ Relay-to-Destination (RD) link, while $h_{i, l}$ is the fading coefficient of the $l^{\text {th }}$ RD link. In Eq. (4), the direct Sourceto-Destination (SD) link is represented by $l=0$, where we have $h_{i, 0}=h_{i, s d}, G_{i, 0}=G_{i, s d}$ and $n_{i, 0}=n_{i, s d}$. Again, it is assumed that the fading and noise variances of the RD links are identical. Meanwhile, $E_{l}$ represents the transmit power at the relay. In order to make the comparison between the equal gain combining technique and the selection combining technique at the destination's receiver convenient, $E_{l}$ is set to $E_{s} \frac{G_{l}}{G_{0}}$. The DS's receiver is illustrated in Fig. 3.

The ReS channel decoders employed at both the RS and the DS rely error-and-erasure decoding in order to improve the achievable performance. In this treatise, our analysis will be focused on the MO-RTT technique, which outperforms both the OTT and the RTT techniques [6], [7].

\section{ReS-Coded System USING ERroR-AND-ERASURE DECODING}

\section{A. At the Relay}

The transmissions from the SS to the relays may be viewed as traditional direct communications between two nodes. Thus, according to [13], the codeword decoding error probability after "errors-and-erasures" $\operatorname{ReS}(N, K)$ decoding at the $l^{\text {th }}$ relay can be expressed as:

$$
P_{w}=\sum_{i=0}^{N} \sum_{j=j_{0}(i)}^{N-i}\left(\begin{array}{c}
N \\
i
\end{array}\right)\left(\begin{array}{c}
N-i \\
j
\end{array}\right) P_{t, l}^{i} P_{e, l}^{j}\left(1-P_{t, l}-P_{e, l}\right)^{N-i-j},
$$

where $j_{0}(i)=\max \{0, N-K+1-2 i\}$, while $P_{e, l}$ and $P_{t, l}$ represent the symbol erasure probability and random symbol error probability before ReS decoding, which are given by Eqs. (38) and (39) of [6] for the MO-RTT.

Hence, the probability of the $l^{t h}$ relay actively forwarding packets to the DS is given by

$$
P_{R f}=1-P_{w} .
$$

\section{B. At the Destination}

1) Symbol Error Probability (SEP): The choice of the diversity combining technique at the destination's receiver significantly affects the achievable SEP performance. For noncoherent detection systems, the EGC and SC are frequently employed. Hence, we will investigate the SEP of these two diversity combining techniques here.

$\boldsymbol{E G C}$ : Based on the assumptions of Section II, the outputs of the low complexity non-coherent square-law detector dispensing with any channel estimates at the DS's receiver may be expressed as

$$
U_{1}=\sum_{l=0}^{L_{f}}\left|G_{l} h_{l} \sqrt{P_{l} R_{c}} e^{-j \phi_{l}}+n_{1, l}\right|^{2},
$$

$$
U_{i}=\sum_{l=0}^{L_{f}}\left|n_{i, l}\right|^{2}, \quad i=2,3, \ldots, M,
$$

where $L_{f}$ is the number of relays forwarding packets to the destination.

According to [14], the p.d.fs of the outputs $U_{1}$ and $U_{i}$ are given as

$$
\begin{array}{r}
f_{U_{1}}(y)=\frac{y^{L_{f}}}{\left(1+\overline{\left.\gamma_{l}\right)^{L_{f}+1} \cdot L_{f} !} \exp \left(-\frac{y}{1+\bar{\gamma}_{l}}\right),\right.} \quad y \geq 0, \\
f_{U_{i}}(y)=\frac{y^{L_{f}}}{L_{f} !} \exp (-y), \quad i=2,3 \ldots M ; \quad y \geq 0,
\end{array}
$$

where we have $\overline{\gamma_{l}}=|\bar{h}|^{2} P_{s} / N_{n}$.

When there are $L_{f}$ relays forwarding packets to the DS, the average erroneous symbol probability of $P_{N_{n}, L_{f}}\left(H_{0}\right)$ at the DS can be expressed as [15]

$$
\begin{aligned}
P_{N_{n}, L_{f}}\left(H_{0}\right)= & 1-\int_{0}^{\infty} f_{U_{1}}(y)\left[\int_{0}^{y} f_{U_{2}}(x) d x\right]^{M-1} d y \\
= & \frac{1}{L_{f} !} \sum_{m=1}^{M-1} \frac{(-1)^{m-1}\left(\begin{array}{c}
M-1 \\
m
\end{array}\right)}{\left(1+m+m \overline{\gamma_{l}}\right)^{L_{f}+1}} \\
& \cdot \sum_{k=0}^{m L_{f}} \beta_{k m}\left(L_{f}+k\right) !\left(\frac{1+\overline{\gamma_{l}}}{1+m+m \overline{\gamma_{l}}}\right)^{k}
\end{aligned}
$$

where $\beta_{k m}$ is the set of coefficients satisfying the following condition

$$
\left(\sum_{k=0}^{L_{f}} \frac{U_{1}^{k}}{k !}\right)^{k}=\sum_{k=0}^{m L_{f}} \beta_{k m} U_{1}^{k} .
$$

$S C$ : The outputs of the low complexity non-coherent squarelaw detector requiring no channel estimates at the DS's receiver may be expressed as

$$
\begin{aligned}
& U_{1}=\max \left\{U_{1, l}\right\}, \quad l=0,1, . ., L_{f}, \\
& U_{i}=\max \left\{U_{i, l}\right\}, \quad i=2,3, \ldots, M,
\end{aligned}
$$

where $U_{1, l}=\left|G_{l} h_{l} \sqrt{P_{l} R_{c}} e^{-j \phi_{l}}+n_{1, l}\right|^{2}$ and $U_{i, l}=\left|n_{i, l}\right|^{2}$.

According to [16], the p.d.fs of the outputs $U_{1}$ and $U_{i}$ are given as

$$
\begin{aligned}
& f_{U_{1}}(y)=\frac{L_{f}+1}{1+\gamma_{l}} \exp \left(-\frac{y}{1+\gamma_{l}}\right)\left[1-\exp \left(-\frac{y}{1+\gamma_{l}}\right)\right]^{L_{f}}, y \geq 0 \\
& f_{U_{i}}(y)=\left(L_{f}+1\right) \exp (-y)[1-\exp (-y)]^{L_{f}}, \quad i=2,3 \ldots M ; \quad y \geq 0 .
\end{aligned}
$$

It also transpired from [16] that when there are $L_{f}$ relays forwarding error-free packets to the DS, the average erroneous symbol probability of $P_{N_{n}, L_{f}}\left(H_{0}\right)$ at the DS can be expressed as

$$
\begin{aligned}
P_{N_{n}, L_{f}}\left(H_{0}\right)= & 1-\sum_{l=1}^{L_{f}+1}(-1)^{l+1}\left(\begin{array}{c}
L_{f}+1 \\
l
\end{array}\right) \\
& \cdot \prod_{m=1}^{\left(L_{f}+1\right)(M-1)} \frac{m}{m+l /\left(1+\gamma_{l}\right)} .
\end{aligned}
$$

SEP: The average erroneous symbol probability of $P_{N_{n}}\left(H_{0}\right)$ at the DS is given by 


$$
P_{N_{n}}\left(H_{0}\right)=\sum_{L_{f}=0}^{L}\left(\begin{array}{c}
L \\
L_{f}
\end{array}\right) P_{R f}^{L_{f}}\left(1-P_{R f}\right)^{L-L_{f}} \cdot P_{N_{n}, L_{f}}\left(H_{0}\right)
$$

while the correct symbol probability is

$$
P_{N_{n}}\left(H_{1}\right)=1-P_{N_{n}}\left(H_{0}\right)
$$

Since the fraction $\rho$ of the band is interfered with, the average erroneous and correct symbol probabilities may finally be expressed as

$$
\begin{aligned}
& P\left(H_{0}\right)=(1-\rho) P_{N_{0}}\left(H_{0}\right)+\rho P_{N_{0}+N_{i} / \rho}\left(H_{0}\right), \\
& P\left(H_{1}\right)=1-P\left(H_{0}\right) .
\end{aligned}
$$

\section{2) Statistics of the Erasure Insertion Related Variables:} Let $\left\{U_{1, l}, U_{2, l}, \ldots, U_{M, l}\right\}$ represent the decision variables input to the MFSK demodulator. We denote the maximum and the "second" maximum of $\left\{U_{1, l}, U_{2, l}, \ldots, U_{M, l}\right\}$ by

$$
\begin{aligned}
& Y_{1}=\max _{1}\left\{U_{1, l}, U_{2, l}, \ldots, U_{M, l}\right\}, \\
& Y_{2}=\max _{2}\left\{U_{1, l}, U_{2, l}, \ldots, U_{M, l}\right\}
\end{aligned}
$$

and the ratio of the "second" maximum to the maximum is formulated as

$$
\lambda=\frac{Y_{2}}{Y_{1}}, \quad 0 \leq \lambda \leq 1
$$

In the context of the joint MO-RTT, the erasure insertion is based on the observation of both the maximum $Y_{1}$ of (22) and the ratio $\lambda$ of (24). In order to erase the low-probability $\operatorname{ReS}$ coded symbols, we assume that $Y_{T}$ and $\lambda_{T}$ are two threshold, which activate an erasure insertion, whenever $Y_{1} \geq Y_{T}$ and $\lambda \geq \lambda_{T}$. Therefore, the joint p.d.fs of $f_{Y_{1}, \lambda}\left(y, r \mid H_{1}\right)$ and $f_{Y_{1}, \lambda}\left(y, r \mid H_{0}\right)$ have to be derived, in order to evaluate the error-and-erasure ReS decoding performance in term of the joint MO-RTT erasure insertion scheme.

$\boldsymbol{E} \boldsymbol{G C}$ : As shown in the Appendix, when EGC is employed, the joint p.d.fs of $Y_{1}$ and $\lambda=Y_{2} / Y_{1}$ under the hypotheses $H_{1}$ of correct decision and $H_{0}$ of erroneous decision, respectively, can be expressed as

$$
\begin{aligned}
f_{Y_{1}, \lambda}\left(y, r \mid H_{1}\right) & \\
= & \frac{(M-1) y}{P_{N_{n}}\left(H_{1}\right)} \cdot \frac{y^{2 L_{f}} r^{L_{f}}}{\left(1+\overline{\gamma_{l}}\right)^{L_{f}+1} \cdot\left(L_{f} !\right)^{M}} e^{\left(-\frac{1+r+r \bar{\gamma}_{l}}{1+\bar{\gamma}_{l}} y\right)} \\
& \cdot\left[L_{f} !-e^{-y r} \sum_{k=0}^{L_{f}} k !\left(\begin{array}{c}
L_{f} \\
k
\end{array}\right)(y r)^{L_{f}-k}\right]^{M-2},
\end{aligned}
$$

and

$$
\begin{aligned}
& f_{Y_{1}, \lambda}\left(y, r \mid H_{0}\right) \\
& =\frac{(M-1) y}{P_{N_{n}}\left(H_{0}\right)} \cdot \frac{y^{2 L_{f}} r^{L_{f}}}{\left(1+\bar{\gamma}_{l}\right)^{L_{f}+1} \cdot\left(L_{f} !\right)^{M}}\left\{e^{\left(-\frac{1+r+r \bar{\gamma}_{l}}{1+\bar{\gamma}_{l}} y\right)}\right. \\
& \quad \cdot\left[L_{f} !-e^{-y r} \sum_{k=0}^{L_{f}} k !\left(\begin{array}{c}
L_{f} \\
k
\end{array}\right)(y r)^{L_{f}-k}\right]^{M-2} \\
& +(M-2) e^{-(r+1) y} \cdot\left[\left(1+\bar{\gamma}_{l}\right)^{L_{f}+1} L_{f} !\right. \\
& \left.\quad-e^{\left(-\frac{y r}{1+\gamma_{l}}\right)} \sum_{k=0}^{L_{f}}\left(1+\bar{\gamma}_{l}\right)^{k+1} k !\left(\begin{array}{c}
L_{f} \\
k
\end{array}\right)(y r)^{L_{f}-k}\right] \\
& \left.\quad \cdot\left[L_{f} !-e^{-y r} \sum_{k=0}^{L_{f}} k !\left(\begin{array}{c}
L_{f} \\
k
\end{array}\right)(y r)^{L_{f}-k}\right]^{M-3}\right\} .
\end{aligned}
$$
are

$$
\begin{aligned}
f_{Y_{1}, \lambda}\left(y, r \mid H_{1}\right) & \frac{(M-1) y}{P_{N_{n}}\left(H_{1}\right)} \cdot \frac{\left(L_{f}+1\right)^{2}}{1+\gamma_{l}} e^{\left(-\frac{1+r+r \gamma_{l}}{1+\gamma_{l}} y\right)} \\
& \cdot\left[1-e^{\left(-\frac{y}{1+\gamma_{l}}\right)}\right]^{L_{f}}\left[1-e^{-y r}\right]^{\left(L_{f}+1\right)(M-2)+L_{f}},
\end{aligned}
$$

and

$$
\begin{aligned}
f_{Y_{1}, \lambda}( & \left.y, r \mid H_{0}\right) \\
= & \frac{(M-1) y}{P_{N_{n}}\left(H_{1}\right)} \cdot\left\{\frac{\left(L_{f}+1\right)^{2}}{1+\gamma_{l}} e^{\left(-\frac{1+r+r \gamma_{l}}{1+\gamma_{l}} y\right)}\right. \\
& \cdot\left[1-e^{\left(-\frac{y}{1+\gamma_{l}}\right)}\right]^{L_{f}}\left[1-e^{-y r}\right]^{\left(L_{f}+1\right)(M-2)+L_{f}} \\
& +(M-2) \cdot \frac{\left(L_{f}+1\right)^{3}}{2} e^{(-y r-y)}\left[1-e^{-y}\right]^{L_{f}} \\
& \cdot\left[1-e^{\left(-\frac{y r}{1+\gamma_{l}}\right)}\right]^{2}\left[1-e^{\left.-y r^{\left(L_{f}+1\right)(M-3)+L_{f}}\right\} .}\right.
\end{aligned}
$$

3) Codeword Error Probability: Based on the statistics formulated above, the symbol erasure probability, $P_{e}$, and the random symbol error probability, $P_{t}$, corresponding to the noise power spectral of $N_{n}$, and the given thresholds of $Y_{T}$ and $\lambda_{T}$, may be expressed as [6]

$$
\begin{aligned}
P_{e}\left(N_{n}, Y_{T}, \lambda_{T}\right)= & P_{N_{n}}\left(H_{1}\right) \int_{0}^{Y_{T}} \int_{\lambda_{T}}^{1} f_{Y_{1}, \lambda}\left(y, r \mid H_{1}\right) d r d y \\
& +P_{N_{n}}\left(H_{0}\right) \int_{0}^{Y_{T}} \int_{\lambda_{T}}^{1} f_{Y_{1}, \lambda}\left(y, r \mid H_{0}\right) d r d y,(29) \\
P_{t}\left(N_{n}, Y_{T}, \lambda_{T}\right)= & P_{N_{n}}\left(H_{0}\right)\left[1-\int_{0}^{Y_{T}} \int_{\lambda_{T}}^{1} f_{Y_{1}, \lambda}\left(y, r \mid H_{0}\right) d r d y\right]
\end{aligned}
$$

Due to the effect of the PBGI, the symbol erasure probability and the random symbol error probability after erasure filling based decoding at the destination may be presented as [6] 


$$
\begin{aligned}
& P_{e}=(1-\rho) P_{e}\left(N_{0}, Y_{T}, \lambda_{T}\right)+\rho P_{e}\left(N_{0}+N_{I} / \rho, Y_{T}, \lambda_{T}\right), \\
& P_{t}=(1-\rho) P_{t}\left(N_{0}, Y_{T}, \lambda_{T}\right)+\rho P_{t}\left(N_{0}+N_{I} / \rho, Y_{T}, \lambda_{T}\right) .
\end{aligned}
$$

As a result, the codeword error probability $P_{w}$ at the destination may be expressed as

$$
P_{w}=\sum_{i=0}^{N} \sum_{j=j_{0}(i)}^{N-i}\left(\begin{array}{c}
N \\
i
\end{array}\right)\left(\begin{array}{c}
N-i \\
j
\end{array}\right) P_{t}^{i} P_{e}^{j}\left(1-P_{t}-P_{e}\right)^{N-i-j}
$$

where $j_{0}(i)=\max \{0, N-K+1-2 i\}$.

Consequently, the symbol error probability $P_{s}$ after errorand-erasure ReS decoding may be expressed as

$P_{s}=\frac{1}{N} \sum_{i=0}^{N} \sum_{j=j_{0}(i)}^{N-i}(i+j)\left(\begin{array}{c}N \\ i\end{array}\right)\left(\begin{array}{c}N-i \\ j\end{array}\right) P_{t}^{i} P_{e}^{j}\left(1-P_{t}-P_{e}\right)^{N-i-j}$

while the bit error probability $P_{b}$ may be approximated as [15]

$$
P_{b}=\frac{2^{b-1}}{M-1} P_{s} .
$$

\section{Performance Results}

Based on the expressions formulated in the previous sections, we will investigate the performance of the lowcomplexity non-coherent FFH MFSK ReS coded systems operating in a cooperative network. For all the performance results below, the channels are assumed to be flat Rayleigh faded. The signal power to interference plus noise ratio (SINR) is set at $10 \mathrm{~dB}$, while the portion of the band, which is interfered by the PBGI, equals 0.15. All relays are assumed to be at the mid-point of the source-to-destination link.

Fig. 4 portrays the relay's codeword error probability at $E_{b} / N_{0}=8 d B$ when utilizing the joint MO-RTT decoding technique. The $\operatorname{Re} S(31,20)$ code and 32-ary FSK modulation are employed. According to the figure, there exists an optimum threshold value of $Y_{T}$ or $\lambda_{T}$, which minimizes the codeword error probability. It is noted that when we have $Y_{T}=0$ or $\lambda_{T}=1$, the error-and-erasure scheme performs similarly to the error-correction-only decoder, or to the scenario, when no erasure insertion is employed. If the threshold value of $Y_{T}$ is excessive and simultaneously, the threshold value of $\lambda_{T}$ is too low, the system will perform worse than the error-correctiononly decoding scheme.

The packet forwarding probability versus $E_{b} / N_{0}$ at the relay is seen in Fig. 5. Three decoding schemes were considered, namely the uncoded scheme, error-correction-only decoding and error-and-erasure decoding. According to Fig. 5, both of the FEC decoding schemes outperform the uncoded scheme. When error-and-erasure decoding is employed, a packet has a higher chance of being forwarded, compared to the errorcorrection-only decoder.

Similar to Fig. 4, the codeword decoding error probability recorded at the destination is shown in Fig. 6 at $E_{b} / N_{0}=$ $8 \mathrm{~dB}$. It is also found from the figure that there is an optimum threshold value of $Y_{T}$ or $\lambda_{T}$, which minimizes the decoding error probability.
Relay's Codeword Error Probability at $\mathrm{E}_{\mathrm{b}} / \mathrm{N}_{0}=8 \mathrm{~dB}$

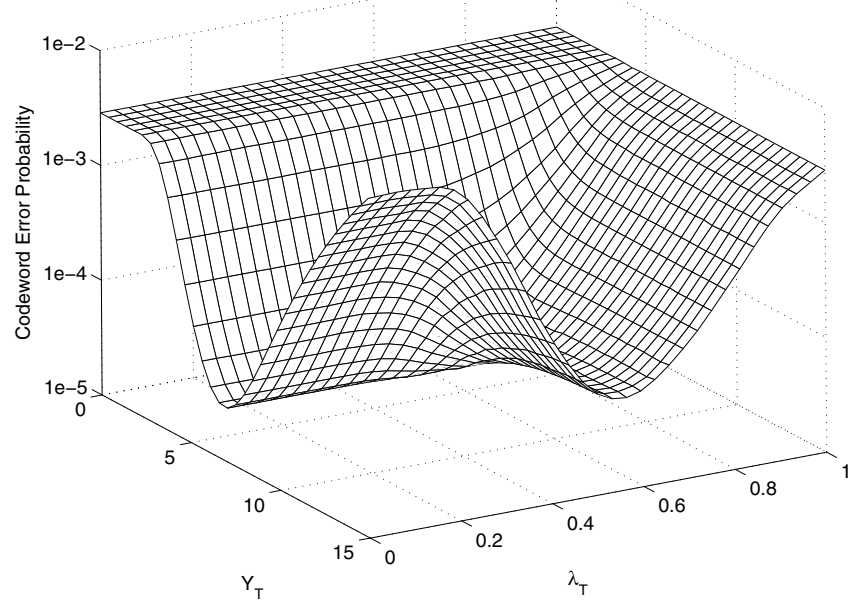

Fig. 4. Codeword decoding error probability at the relay versus the amplitude threshold, $Y_{T}$ and the ratio threshold, $\lambda_{T}$ for the $\operatorname{ReS}(31,20)$ FEC code using "error-and-erasure" decoding based on the MO-RTT erasure insertion scheme for transmission over a flat Rayleigh fading channel: $\rho=0.15, M=$ $32, E_{b} / N_{0}=8 d B, E_{b} / N_{I}=10 d B, \operatorname{Re} S(31,20), L=1, G_{s r}=G_{r d}=$ $4 G_{s d}$.

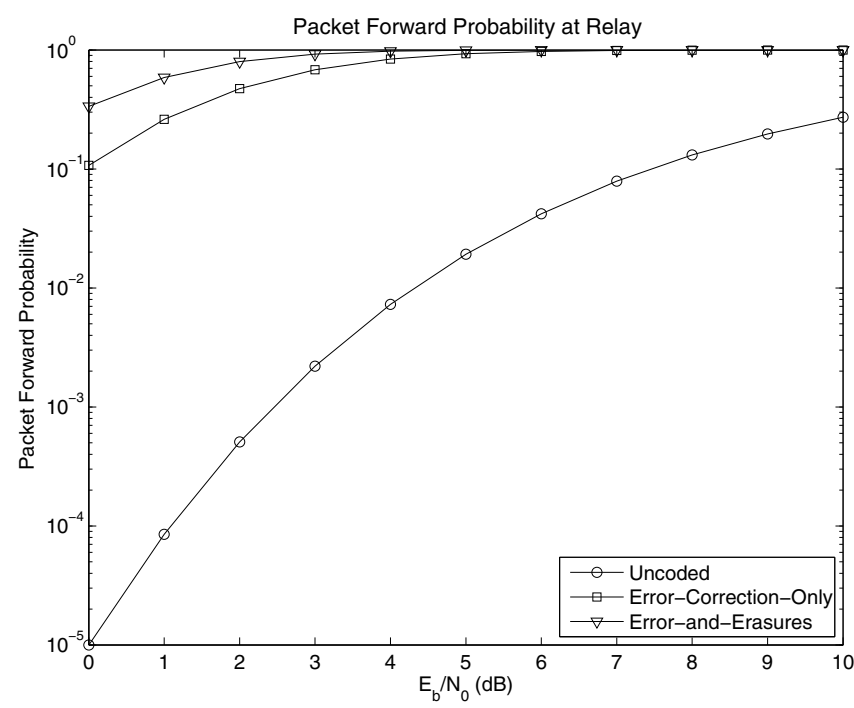

Fig. 5. Packet forward probability at the relay for the $\operatorname{ReS}(31,20)$ FEC code using "error-correction-only" decoding and "error-and-erasure" decoding based on the MO-RTT erasure insertion scheme for transmission over a flat Rayleigh fading channel. The results were evaluated from (6): $\rho=$ $0.15, M=32, E_{b} / N_{I}=10 d B, \operatorname{Re} S(31,20), L=1, G_{s r}=G_{r d}=$ $4 G_{s d}$.

Fig. 7 compared the achievable performance of the erasure filling and non-erasure-filling decoding schemes for different FSK/ReS schemes. Naturally, the erasure insertion schemes outperform the non-erasure-insertion ones. When employing the $\operatorname{Re} S(31,20)$ code combined with 32-FSK modulation and relying on a single relay, the error-and-erasure decoding scheme achieves a $3 \mathrm{~dB} E_{b} / N_{0}$ gain at Codeword Error Probability, $P_{w}$, of $10^{-4}$, compared to the error-correctiononly scheme. Moreover, the ReS code, which employs a highorder Galois field, combined with high-order FSK modulation, 


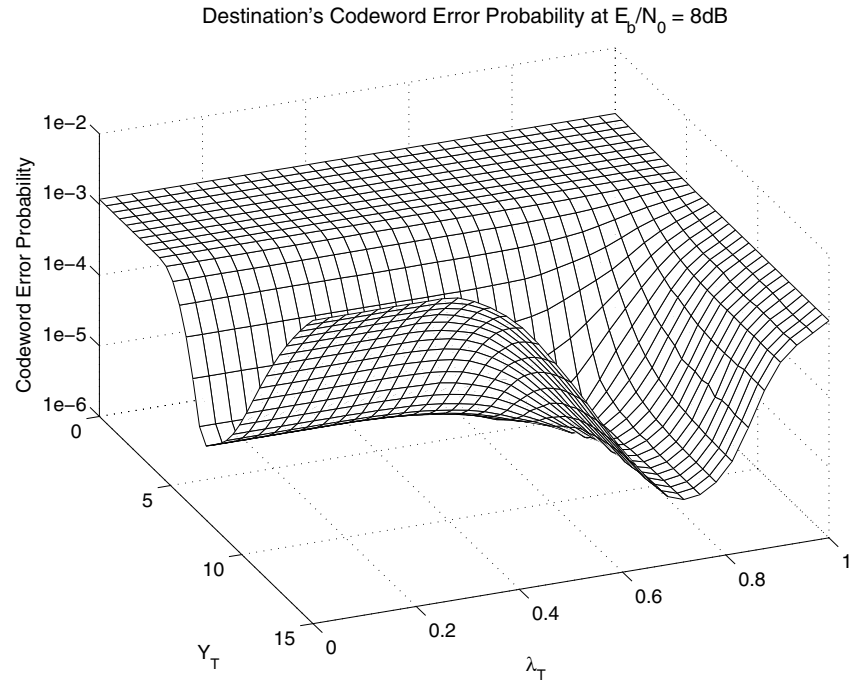

Fig. 6. Codeword decoding error probability at the destination versus the amplitude threshold, $Y_{T}$ and the ratio threshold, $\lambda_{T}$ for the $\operatorname{ReS}(31,20)$ FEC code using "error-and-erasure" decoding based on the MO-RTT erasure insertion scheme for transmission over a flat Rayleigh fading channel. The results were evaluated from (33): $\rho=0.15, M=32, E_{b} / N_{0}=8 d B, E_{b} / N_{I}=$ $10 d B, \operatorname{Re} S(31,20), L=1, G_{s r}=G_{r d}=4 G_{s d}$.

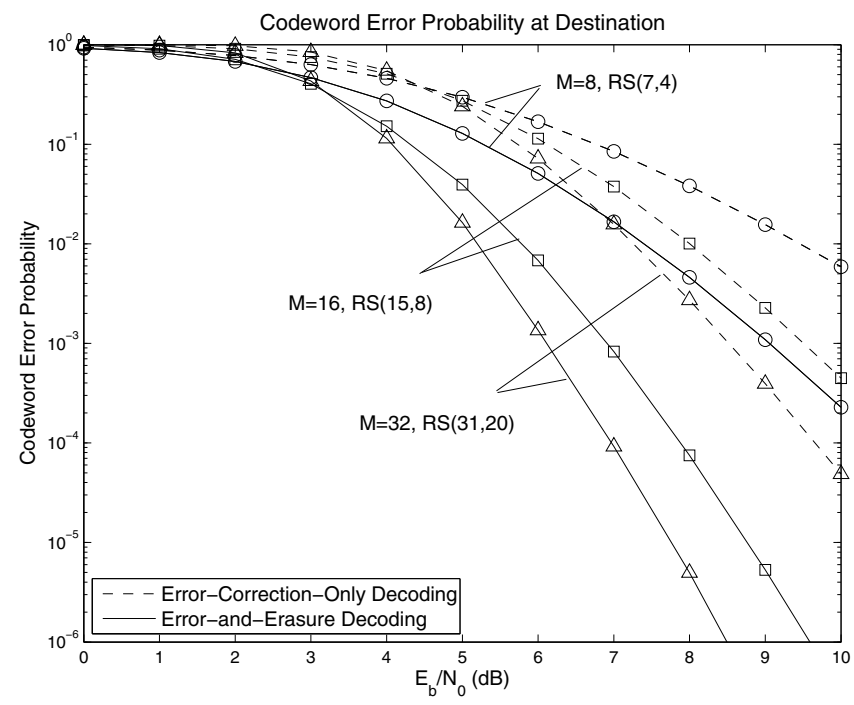

Fig. 7. Codeword decoding error probability at the destination for different ReS FEC codes using "error-correction-only" decoding and "errorand-erasure" decoding based on the MO-RTT erasure insertion scheme for transmission over a flat Rayleigh fading channel. The results were evaluated from (33): $\rho=0.15, E_{b} / N_{I}=10 d B, L=1, G_{s r}=G_{r d}=4 G_{s d}$.

perform significantly better above $5 \mathrm{~dB}$, than those using lowerorder Galois field combined with low-order FSK modulation.

The system performances of the EGC and SC techniques are compared in Fig. 8. According to the figure, the EGC schemes achieved a lower codeword error probability than the SC arrangement. More particularly, the EGC scheme may achieve a $1.5 \mathrm{~dB}$ gain at $P_{w}=10^{-6}$, compared to the SC scheme, when assisted by a single relay. Likewise, the attainable $E_{b} / N_{0}$ gain is $1.0 \mathrm{~dB}$, when there are two assisting relays. This is not unexpected, because the EGC technique constitutes the optimal diversity combining scheme for non-

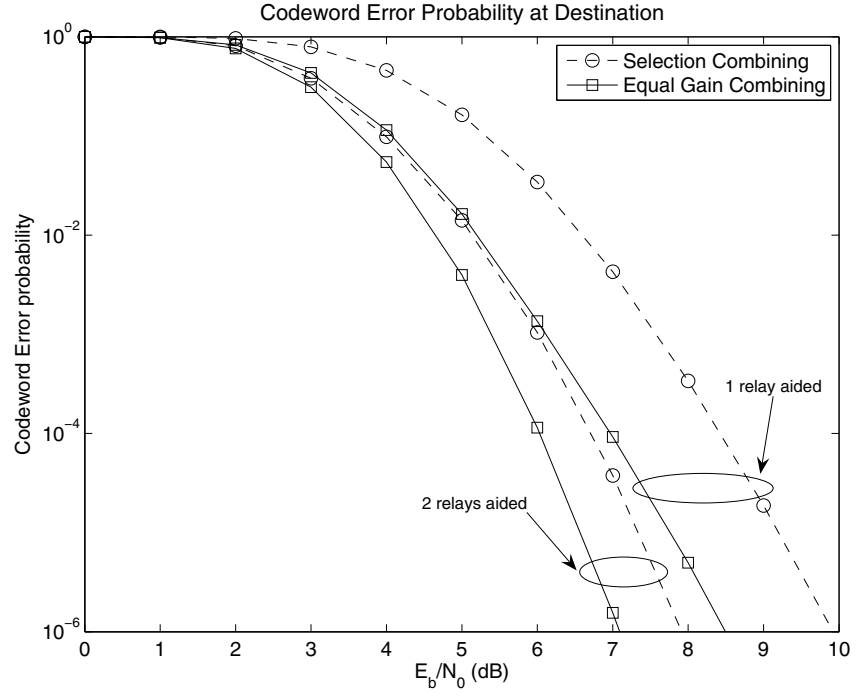

Fig. 8. Codeword decoding error probability at the destination for the $\operatorname{ReS}(31,20)$ FEC codes using "error-and-erasure" decoding based on the MORTT erasure insertion scheme combined with the EGC and SC techniques for transmission over a flat Rayleigh. The results were evaluated from (33): $\rho=0.15, M=32, E_{b} / N_{I}=10 d B, \operatorname{Re} S(31,20), L=1, G_{s r}=G_{r d}=$ $4 G_{s d}$.

coherent detection.

The beneficial effect of the number of relays is shown in Fig. 9. Observe that below $E_{b} / N_{0}$ of $2 \mathrm{~dB}$, the schemes relying on a higher number of relays might in fact perform worse than those relying on a lower number of relays. This is because the total available power is distributed between the source and the relays for the sake of a fair comparison. As a result, the transmit power of both the source as well as the relays may become insufficient for their successful decoding and hence, the packet forward probability at the relay decreased. In other words, the number of relays forwarding data to the destination is reduced. When the $E_{b} / N_{0}$ value increases, more relays forward data to the destination. Thus, the schemes associated with a higher number of relays perform better than those relying on less relays. However, the BER gain associated with employing more than two relays gradually becomes insignificant upon increasing the number of relays. The attainable performance becomes similar to that of the colocated MIMO system upon increasing the number of transmit antennas.

The effects of interference are characterized in Fig. 10, when the partial-band interference fraction $\rho$ is varied. As expected, the codeword error probability increases as a function of $\rho$, i.e when imposing more interference on the signal's band. More particularly, at $E_{b} / N_{0}$ of $7 \mathrm{~dB}$ and $E_{b} / N_{I}$ of $5 \mathrm{~dB}, P_{W}$ increases from $10^{-4}$ to 0.5 when $\rho$ is increased from 0.1 to 0.9 .

Furthermore, we validate the performance of the systems subjected to theoretical analysis in Section III by computer simulations. The results of Fig. 11 showed that there is a slight gap of approximately $0.5 \mathrm{~dB}$ between the analysis and simulations. This can be explained by the fact that the theoretical analysis employs some simplifying approximations, such as those applied to the p.d.fs of the detector's outputs [6], 


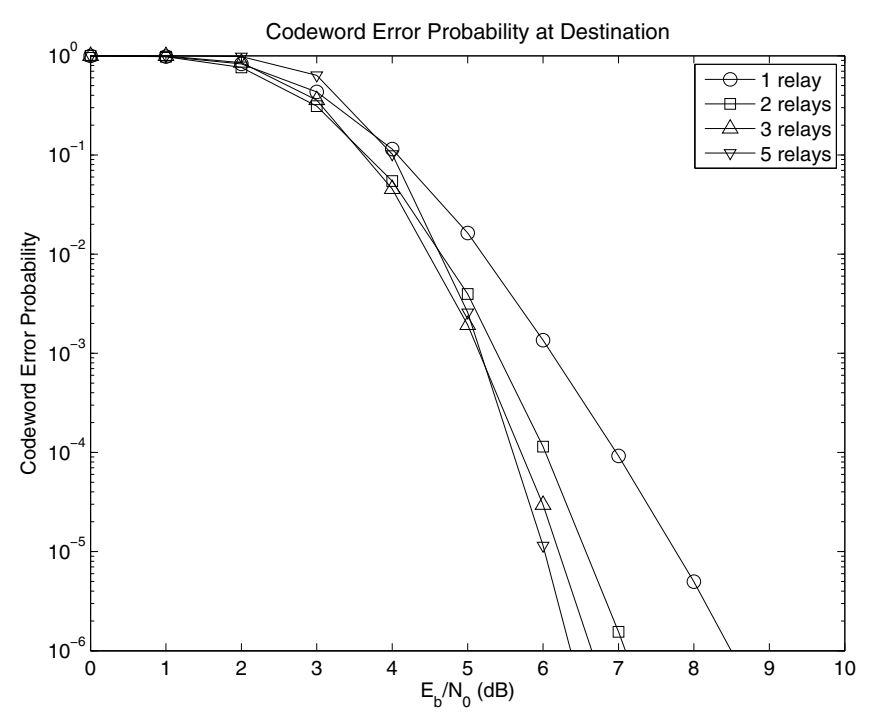

Fig. 9. Codeword decoding error probability at the destination for the $\operatorname{ReS}(31,20)$ FEC codes using "error-and-erasure" decoding based on the MORTT erasure insertion scheme for transmission over a flat Rayleigh fading channel when varying the number of relays. The results were evaluated from (33): $\rho=0.15, M=32, E_{b} / N_{I}=10 d B, \operatorname{Re} S(31,20), L=$ $\{1,2,3,5\}, G_{s r}=G_{r d}=4 G_{s d}$.

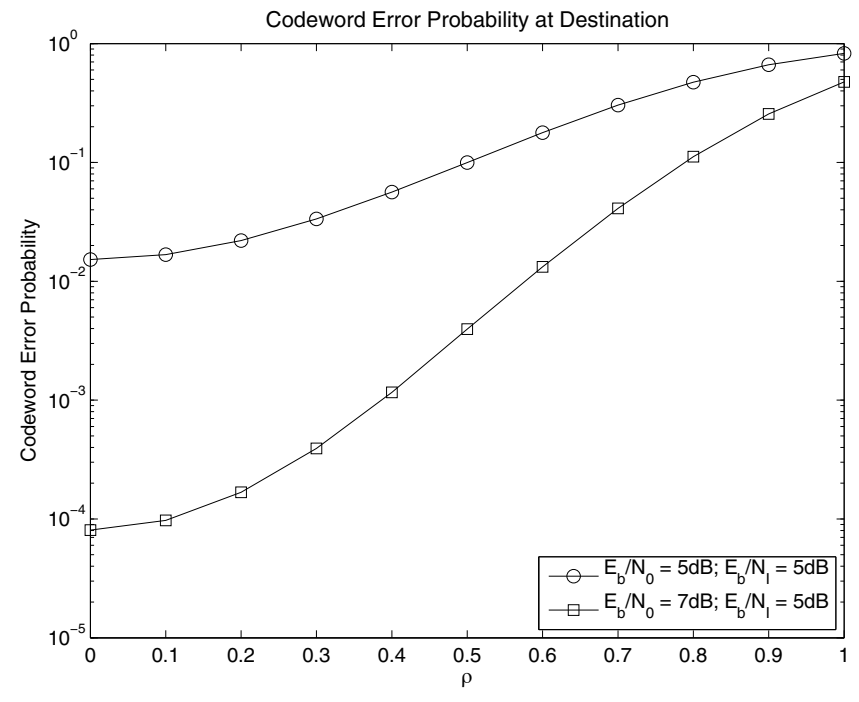

Fig. 10. Codeword decoding error probability at the destination for the $\operatorname{ReS}(31,20)$ FEC codes using "error-and-erasure" decoding based on the MORTT erasure insertion scheme for transmission over a flat Rayleigh fading channel when varying the interference fraction $\rho$. The results were evaluated from (33): $M=32, \operatorname{Re} S(31,20), L=\{1,2,3,5\}, G_{s r}=G_{r d}=4 G_{s d}$.

[14], which are given in Eqs. $(9,10,15,16)$ or to the average erroneous symbols probability of Eq. (11) [15]. Additionally, as seen in Figs. 4 and 6, the CEP depends on the values of the thresholds, $Y_{T}$ and $\lambda_{T}$. For a given received SNR, there is an optimum pair of $Y_{T}$ and $\lambda_{T}$ values, which provides the lowest CEP. In the analysis we use the average received SNR for evaluating the CEP, while in the simulations, a given pair of $Y_{T}$ and $\lambda_{T}$ values is used for all the instantaneous received SNRs, which varied in accordance with the channel coefficients. Therefore, the simulation results are not perfectly accurate either.

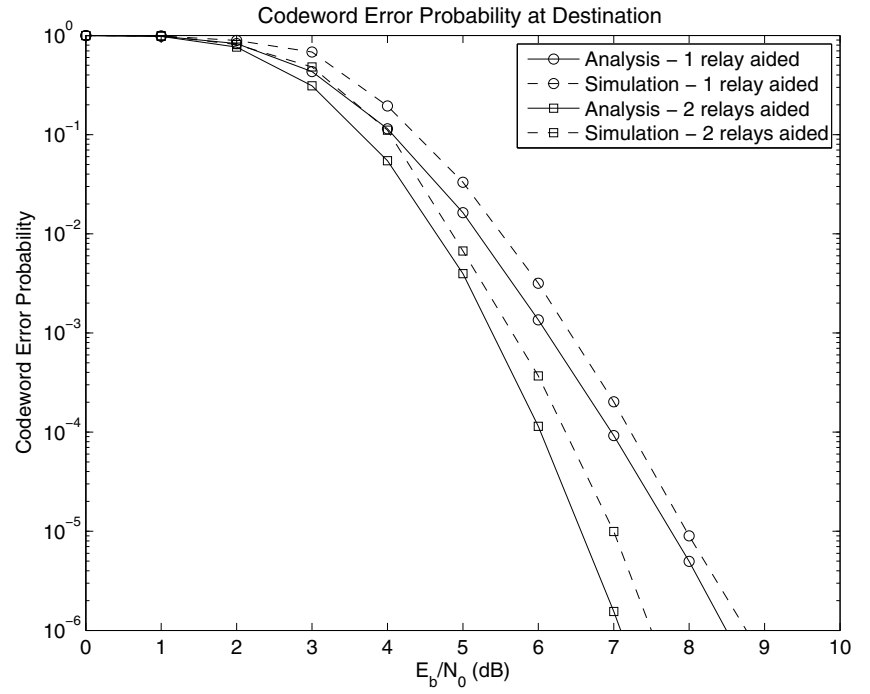

Fig. 11. Codeword decoding error probability at the destination for the $\operatorname{ReS}(31,20)$ FEC codes using "error-and-erasure" decoding based on the MO-RTT erasure insertion scheme for transmission over a flat Rayleigh fading channel. The results were achieved by simulation and from (33): $\rho=0.15, M=32, E_{b} / N_{I}=10 d B, \operatorname{Re} S(31,20), L=\{1,2\},, G_{s r}=$ $G_{r d}=4 G_{s d}$.

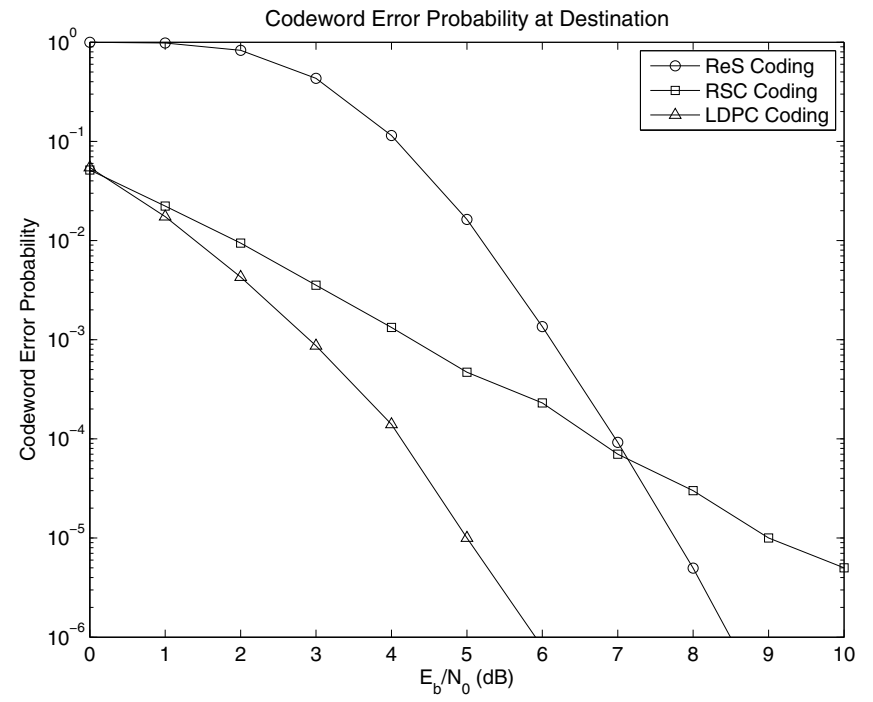

Fig. 12. Codeword decoding error probability at the destination for the RSC $(23,33)$ code, LDPC codes and the ReS $(31,20)$ FEC code using "errorand-erasure" decoding based on the MO-RTT erasure insertion scheme for transmission over a flat Rayleigh fading channel when varying the interference fraction $\rho$. The results were evaluated from (33): $M=32, \operatorname{Re} S(31,20), L=$ $\{1,2,3,5\}, G_{s r}=G_{r d}=4 G_{s d}$.

Finally, we compare the performance of the proposed ReS coded scheme using error-and-erasure decoding to that of other coding schemes, such as the classic convolutional and LDPC codes in the context of wireless cooperative networks. The Recursive Systematic Covoluational (RSC) code, RSC $(23,33)$, using the octally represented generator polynomials of 23 and 33 [15] is considered and a regular LDPC code [10] is employed. For a fair comparison, the same coding rate and the packet size are employed for all three coding schemes. For the RSC code and the LDPC code, iterative detection is 
employed at the cost of a potentially high complexity and latency imposed on the systems. As seen in Fig. 12, the LDPC coded scheme outperforms both the ReS coded and the RSC coded scheme. More particularly, it achieves a power gain of $3 \mathrm{~dB}$ compared to the ReS coded scheme at the CEP of $10^{-6}$. By contrast, the RSC coded scheme performs better than the ReS coded scheme only in the low $E_{b} / N_{0}$ region, namely below $7 \mathrm{~dB}$ and no turbo cliff exists even when iterative detection is employed for the RSC scheme. This may be explained by the fact that the iterative detection aided RSC codes only performs well, when the packet size is long, which is not the case in the cooperative scenario considered.

\section{CONCLusions}

In this paper, we have proposed a joint MO-RTT erasure insertion aided ReS coded and non-coherently detected SFH MFSK system for cooperative networks. The corresponding mathematical expressions were derived in order to quantify the attainable performance of the proposed system. The results of Fig. 7 showed that the error-and-erasure ReS decoding scheme outperforms the error-correction-only ReS decoding arrangement. More particularly, the $\operatorname{Re} S(31,20)$ coded system combined with 32-FSK modulation employing error-and-erasure decoding may achieve an $E_{b} / N_{0}$ gain of approximately $3 \mathrm{~dB}$ at $P_{w}$ of $10^{-4}$, compared to the same system using errorcorrection-only decoding. Moreover, we have compared the achievable performance of the system, when employing the EGC and SC techniques at the receiver. It was found in Fig. 8 that the EGC scheme assisted by one and two relays, is capable of achieving an $E_{b} / N_{0}$ gain of $1.5 \mathrm{~dB}$ and $1.0 \mathrm{~dB}$, respectively, at $P_{w}$ of $10^{-6}$, compared to the SC scheme. Finally, we demonstrated that the performance of the proposed ReS coded scheme is inferior to that of the LDPC codes considered, but it is capable of outperforming convolutional codes, when short packet transmissions are considered.

\section{APPENDIX}

In this appendix we drive the joint conditional p.d.fs of $Y_{1}$ and $\lambda=Y_{2} / Y_{1}$ in the context of the MO-RTT, when the relay-aided SFH-MFSK system operates in flat Rayleigh fading channels.

According to [6], the joint conditional p.d.fs $f_{Y_{1}, \lambda}\left(y, r \mid H_{1}\right)$ and $f_{Y_{1}, \lambda}\left(y, r \mid H_{0}\right)$ associated with the MO-RTT based erasure insertion scheme may be expressed as

$$
\begin{aligned}
& f_{Y_{1}, \lambda}\left(y, r \mid H_{1}\right)= \\
& \quad \frac{(M-1) y}{P_{N_{n}}\left(H_{1}\right)} f_{U_{1}}(y) f_{U_{m}}(y r)\left[\int_{0}^{y r} f_{U_{j}}(x) d x\right]^{M-2}, \\
& 0 \leq y<\infty, 0 \leq r \leq 1
\end{aligned}
$$

$$
\begin{aligned}
& f_{Y_{1}, \lambda}\left(y, r \mid H_{0}\right)= \\
& \quad \frac{(M-1) y}{P_{N_{n}}\left(H_{0}\right)}\left\{f_{U_{m}}(y) f_{U_{1}}(y r)\left[\int_{0}^{y r} f_{U_{j}}(x) d x\right]^{M-2}\right. \\
& \quad+(M-2) f_{U_{m}}(y) f_{U_{j}}(y r)\left[\int_{0}^{y r} f_{U_{1}}(x) d x\right] \\
& \left.\quad \cdot\left[\int_{0}^{y r} f_{U_{k}}(x) d x\right]^{M-3}\right\}, \quad 0 \leq y<\infty, 0 \leq r \leq 1,
\end{aligned}
$$

where $f_{U_{1}}(y)$ represents the p.d.f of (9) and (15), while, $f_{U_{m}}(y), f_{U_{j}}(y)$ and $f_{U_{k}}(y)$ represent the p.d.f of (10) and (16).

\section{A. Equal Gain Combining}

Substituting (9) and (10) into Eqs. (36) and (37) above and employing the function (2.321.2) of [17], we have

$$
\begin{aligned}
f_{Y_{1}, \lambda}\left(y, r \mid H_{1}\right) \\
=\frac{(M-1) y}{P_{N_{n}}\left(H_{1}\right)} \cdot \frac{y^{2 L_{f}} r^{L_{f}}}{\left(1+\overline{\gamma_{l}}\right)^{L_{f}+1} \cdot\left(L_{f} !\right)^{2}} \\
\quad \cdot e^{\left(-\frac{1+r+r \overline{\gamma_{l}}}{1+\bar{\gamma}_{l}} y\right)\left[\int_{0}^{y r} \frac{x^{L_{f}}}{L_{f} !} e^{-x} d x\right]^{M-2}} \\
=\frac{(M-1) y}{P_{N_{n}}\left(H_{1}\right)} \cdot \frac{y^{2 L_{f}} r^{L_{f}}}{\left(1+\overline{\gamma_{l}}\right)^{L_{f}+1} \cdot\left(L_{f} !\right)^{M}} e^{\left(-\frac{1+r+r \bar{\gamma}_{l}}{1+\gamma_{l}} y\right)} \\
\quad \cdot\left[L_{f} !-e^{-y r} \sum_{k=0}^{L_{f}} k !\left(\begin{array}{c}
L_{f} \\
k
\end{array}\right)(y r)^{L_{f}-k}\right]^{M-2},
\end{aligned}
$$

and

$$
\begin{aligned}
& f_{Y_{1}, \lambda}\left(y, r \mid H_{0}\right) \\
& =\frac{(M-1) y}{P_{N_{n}}\left(H_{0}\right)}\left\{\frac{y^{2 L_{f}} r^{L_{f}}}{\left(1+\bar{\gamma}_{l}\right)^{L_{f}+1} \cdot\left(L_{f} !\right)^{2}} e^{\left(-\frac{r+1+\bar{\gamma}_{l}}{1+\bar{\gamma}_{l}} y\right)}\right. \\
& \cdot\left[\int_{0}^{y r} \frac{x^{L_{f}}}{L_{f} !} e^{-x} d x\right]^{M-2}+(M-2) \frac{y^{2 L_{f}} r^{L_{f}}}{\left(L_{f} !\right)^{2}} e^{-(r+1) y} \\
& \cdot\left[\int_{0}^{y r} \frac{x^{L_{f}}}{\left(1+\bar{\gamma}_{l}\right)^{L_{f}+1} \cdot L_{f} !} e^{\left(-\frac{x}{1+\gamma_{l}}\right)} d x\right] \\
& \left.\cdot\left[\int_{0}^{y r} \frac{x^{L_{f}}}{L_{f} !} e^{-x} d x\right]^{M-3}\right\} \\
& =\frac{(M-1) y}{P_{N_{n}}\left(H_{0}\right)} \cdot \frac{y^{2 L_{f}} r^{L_{f}}}{\left(1+\bar{\gamma}_{l}\right)^{L_{f}+1} \cdot\left(L_{f} !\right)^{M}}\left\{e^{\left(-\frac{1+r+r \bar{\gamma}_{\bar{\gamma}}}{1+\bar{\gamma}_{l}} y\right)}\right. \\
& \cdot\left[L_{f} !-e^{-y r} \sum_{k=0}^{L_{f}} k !\left(\begin{array}{c}
L_{f} \\
k
\end{array}\right)(y r)^{L_{f}-k}\right]^{M-2} \\
& +(M-2) e^{-(r+1) y} \cdot\left[\left(1+\bar{\gamma}_{l}\right)^{L_{f}+1} L_{f} !\right. \\
& \left.-e^{\left(-\frac{y r}{1+\bar{\gamma}_{l}}\right)} \sum_{k=0}^{L_{f}}\left(1+\overline{\gamma_{l}}\right)^{k+1} k !\left(\begin{array}{c}
L_{f} \\
k
\end{array}\right)(y r)^{L_{f}-k}\right] \\
& \left.\cdot\left[L_{f} !-e^{-y r} \sum_{k=0}^{L_{f}} k !\left(\begin{array}{c}
L_{f} \\
k
\end{array}\right)(y r)^{L_{f}-k}\right]^{M-3}\right\} \text {. }
\end{aligned}
$$

and 


\section{B. Selection Combining}

Substituting (15) and (16) into Eqs. (36) and (37), we have

$$
\begin{aligned}
& f_{Y_{1}, \lambda}\left(y, r \mid H_{1}\right) \\
&=\frac{(M-1) y}{P_{N_{n}}\left(H_{1}\right)} \cdot \frac{L_{f}+1}{1+\gamma_{l}} e^{\left(-\frac{y}{1+\gamma_{l}}\right)}\left[1-e^{\left(-\frac{y}{1+\gamma_{l}}\right)}\right]^{L_{f}} \\
& \cdot\left(L_{f}+1\right) e^{(-y r)}\left[1-e^{(-y r)}\right]^{L_{f}} \\
& \cdot\left[\int_{0}^{y r}\left(L_{f}+1\right) e^{-x}\left(1-e^{-x}\right)^{L_{f}} d x\right]^{M-2} \\
&=\frac{(M-1) y}{P_{N_{n}}\left(H_{1}\right)} \cdot \frac{\left(L_{f}+1\right)^{2}}{1+\gamma_{l}} e^{\left(-\frac{1+r+r \gamma_{l}}{1+\gamma_{l}} y\right)} \\
& \cdot\left[1-e^{\left(-\frac{y}{1+\gamma_{l}}\right)}\right]^{L_{f}}\left[1-e^{-y r^{\left(L_{f}+1\right)(M-2)+L_{f}},}\right.
\end{aligned}
$$

and

$$
\begin{aligned}
f_{Y_{1}, \lambda}( & \left.y, r \mid H_{0}\right) \\
= & \frac{(M-1) y}{P_{N_{n}}\left(H_{0}\right)}\left\{\left(L_{f}+1\right) e^{-y}\left(1-e^{-y}\right)^{L_{f}} \cdot \frac{L_{f}+1}{1+\gamma_{l}}\right. \\
\cdot & e^{\left(-\frac{y r}{1+\gamma_{l}}\right)}\left[1-e^{\left.\left(-\frac{y r}{1+\gamma_{l}}\right)\right]^{L_{f}}\left[1-e^{(-y r)}\right]^{\left(L_{f}+1\right)(M-2)}}\right. \\
+ & (M-2) \cdot\left(L_{f}+1\right) e^{-y}\left(1-e^{-y}\right)^{L_{f}}\left(L_{f}+1\right) \\
& \cdot e^{(-y r)}\left[1-e^{(-y r)}\right]^{L_{f}} \cdot\left[1-e^{(-y r)}\right]^{\left(L_{f}+1\right)(M-3)} \\
& \left.\cdot \int_{0}^{y r} \frac{L_{f}+1}{1+\gamma_{l}} e^{\left(-\frac{x}{1+\gamma_{l}}\right)}\left[1-e^{\left(-\frac{x}{1+\gamma_{l}}\right)}\right] d x\right\} \\
= & \frac{(M-1) y}{P_{N_{n}}\left(H_{1}\right)} \cdot\left\{\frac{\left(L_{f}+1\right)^{2}}{1+\gamma_{l}} e^{\left(-\frac{1+r+r \gamma_{l}}{1+\gamma_{l}} y\right)}\right. \\
& \cdot\left[1-e^{\left.\left(-\frac{y}{1+\gamma_{l}}\right)\right]^{L_{f}}\left[1-e^{-y r}\right]^{\left(L_{f}+1\right)(M-2)+L_{f}}}\right. \\
& +(M-2) \cdot \frac{\left(L_{f}+1\right)^{3}}{2} e^{(-y r-y)}\left[1-e^{-y}\right]^{L_{f}} \\
& \cdot\left[1-e^{\left.\left.\left(-\frac{y r}{1+\gamma_{l}}\right)\right]^{2}\left[1-e^{-y r}\right]^{\left(L_{f}+1\right)(M-3)+L_{f}}\right\}}\right.
\end{aligned}
$$

\section{REFERENCES}

[1] L. Hanzo, T. H. Liew, B. L. Yeap, R. Y. S. Tee, and S. X. Ng, Turbo Coding, Turbo Equalisation and Space-Time Coding: EXIT-Chart-Aided Near-Capacity Designs for Wireless Channels, 2nd edition. John Wiley \& Sons, Inc., 2011.

[2] L. Hanzo, O. Alamri, M. El-Hajjar, and N. Wu, Near-Capacity MultiFunctional MIMO Systems: Sphere-Packing, Iterative Detection and Cooperation. John Wiley - IEEE Press, May 2009.

[3] Information Technology-Telecommunications and Information Exchange between Systems-Local and Metropolitan Area NetworksSpecific Requirements Part 11: Wireless LAN Medium Access Control (MAC) and Physical Layer (PHY) Specifications Amendment 5: Enhancements for Higher Throughput, IEEE Standard, 2009.

[4] A. Sendonaris, E. Erkip, and B. Aazhang, "User cooperation diversitypart I: system description," IEEE Trans. Commun., vol. 51, pp. 19271938, Nov. 2003

[5] A. Sendonaris, E. Erkip, and B. Aazhang, "User cooperation diversitypart II: implementation aspects and performance analysis," IEEE Trans. Commun., vol. 51, pp. 1939-1948, Nov. 2003.

[6] L.-L. Yang and L. Hanzo, "Low complexity erasure insertion in RScoded SFH spread-spectrum communications with partial-band interference and Nakagami-m fading," IEEE Trans. Commun., vol. 50, pp. 914 925, June 2002.

[7] S. Ahmed, L.-L. Yang, and L. Hanzo, "Erasure insertion in RS-Coded SFH MFSK subjected to tone jamming and Rayleigh fading," IEEE Trans. Veh. Technol., vol. 56, pp. 3563-3571, Nov. 2007.
[8] L. Hanzo, L.-L. Yang, E.-L. Kuan, and K. Yen, Single and Multi-Carrier DS-CDMA: Multi-User Detection, Space-Time Spreading, Synchronisation, Networking and Standards. Wiley-IEEE Press, 2003.

[9] A. J. Viterbi, "A robust ratio-threshold technique to mitigate tone and partial band jamming in coded MFSK systems," in Proc. 1982 IEEE Military Commun. Conf., vol. 1, pp. 22.4-1 - 22.4-5, 17-20.

[10] R. G. Gallager, Low-Density Parity-Check Codes. MIT Press, 1963.

[11] R. Steele and L. Hanzo, Mobile Radio Communications: Second and Third-Generation Cellular and WATM Systems, 2nd edition. John Wiley - IEEE Press, 1999.

[12] H. Ochiai, P. Mitran, and V. Tarokh, "Design and analysis of collaborative diversity protocols for wireless sensor networks," in Proc. 2004 IEEE Veh. Technol. Conf. - Fall, vol. 7, pp. 4645-4649.

[13] C. Baum and M. Pursley, "Bayesian methods for erasure insertion in frequency-hop communication systems with partial-band interference," IEEE Trans. Commun., vol. 40, pp. 1231-1238, July 1992.

[14] P. Hahn, "Theoretical diversity improvement in multiple frequency shift keying," IRE Trans. Commun. Syst., vol. 10, pp. 177-184, June 1962.

[15] J. G. Proakis, Digital Communications, 4th edition. McGraw-Hill, 2001.

[16] L.-L. Yang, K. Yen, and L. Hanzo, "A Reed-Solomon coded DS-CDMA system using noncoherent M-ary orthogonal modulation over multipath fading channels," IEEE J. Sel. Areas Commun., vol. 18, pp. 2240-2251, Nov. 2000.

[17] I. S. Gradshteyn, A. Jeffrey, and I. M. Ryzhik, Table of Integrals, Series, and Products, 7th edition. Academic Press, 2007.

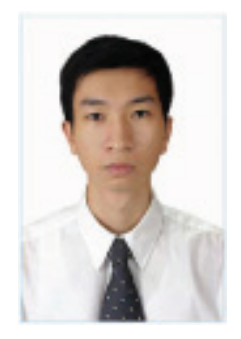

Hoang Anh Ngo received his BEng degree (with first class honour) in electronic engineering from Hanoi University of Science and Technology (HUST), Vietnam, and his MSc degree (with distinction) in wireless communications from the University of Southampton, UK. Since November 2008, he has been working towards his Ph.D. degree with the Communications, Signal Processing and Control (CSPC) group, School of Electronics and Computer Science, University of Southampton, UK. $\mathrm{He}$ is the recipient of several academic awards from HUST and the University of Southampton, as well as the Engineering and Physical Sciences Research Council (EPSRC), UK. His research interests include co-located and distributed MIMO communications, space-time coding and modulation, channel modelling, and cross-layer optimization.

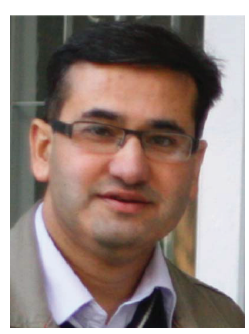

Sohail Ahmed earned his Ph.D. from the University of Southampton, UK, in 2007. His research interests include frequency hopping systems, noncoherent modulation schemes, diversity combining, and software defined radio. He is currently working as an associate professor at the National University of Sciences and Technology, Pakistan.

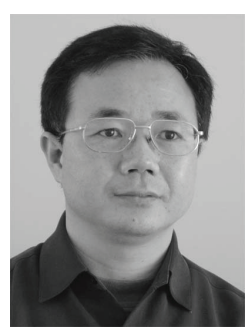

Lie-Liang Yang (M'98-SM'02) received his BEng degree in communications engineering from Shanghai TieDao University, Shanghai, China in 1988, and his MEng and Ph.D. degrees in communications and electronics from Northern (Beijing) Jiaotong University, Beijing, China, in 1991 and 1997, respectively. From June 1997 to December 1997, he was a visiting scientist at the Institute of Radio Engineering and Electronics, Academy of Sciences of the Czech Republic. Since December 1997, he has been with the University of Southampton, United Kingdom, where he is a professor of wireless communications in the School of Electronics and Computer Science. Dr. Yang's research has covered a wide range of topics in wireless communications, networking, and signal processing. He has published over 270 research papers in journals and conference proceedings, authored/co-authored three books, and also published several book chapters. He is currently an associate editor to the IEEE TRANSACTIONS ON VEHICULAR TECHNOLOGY, the Journal of Communications and Networks (JCN), and the Security and Communication Networks (SCN) Journal. 


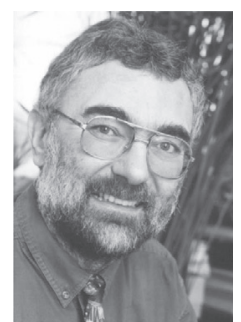

Lajos Hanzo (FREng, FIEEE, FIET, Fellow of EURASIP, DSc) received his degree in electronics in 1976 and his doctorate in 1983. In 2009, he was awarded the honorary doctorate "Doctor Honaris Causa" by the Technical University of Budapest. During his 35-year career in telecommunications, he has held various research and academic posts in Hungary, Germany, and the UK. Since 1986, he has been with the School of Electronics and Computer Science, University of Southampton, UK, where he holds the chair in telecommunications. He has successfully supervised in excess of $70 \mathrm{PhD}$ students, co-authored 20 John Wiley/IEEE Press books on mobile radio communications totalling in excess of 10000 pages, published 1200+ research entries at IEEE Xplore, acted both as TPC and General Chair of IEEE conferences, presented keynote lectures, and has been awarded a number of distinctions.

Currently he is directing an academic research team, working on a range of research projects in the field of wireless multimedia communications, sponsored by industry, the Engineering and Physical Sciences Research Council (EPSRC), UK, the European IST Programme, and the Mobile Virtual Centre of Excellence (VCE), UK. He is an enthusiastic supporter of industry and an academic liaison, and he offers a range of industrial courses. He is also a Governor of the IEEE VTS. Since 2008, he has been the Editor-inChief of the IEEE Press and since 2009 a Chaired Professor, also at Tsinghua University, Beijing. In 2012, he became one of four EURASIP Fellows. For further information on research in progress and associated publications please refer to http://www-mobile.ecs.soton.ac.uk 